\title{
3.0T 1H MRS in Assessing the Steatosis in Chronic Hepatitis C Patients
}

\author{
Qian Zhang, Yonggui Zhang, Ping Zhao, Jian Jiao, Jiangbin Wang, Wenqian Qi \\ Department of Gastroenterology, China-Japan Union Hospital Affiliated to Jilin University, Changchun, Jilin, \\ China
}

Keywords: $1 \mathrm{H}$; MRS; hepatitis C; antiviral therapy.

\begin{abstract}
To investigate the utility of $1 \mathrm{H}$ magnetic resonance spectroscopy (MRS) as a noninvasive test for steatosis in patients infected with hepatitis C virus (HCV), ninety patients with chronic hepatitis $\mathrm{C}$ and pathology data underwent 3.0T 1H MRS, and the results of MRS and pathological analysis were compared. Results show that this group of patients included 26 people with mild fatty liver (28.89\%), 16 people with moderate fatty liver (17.78\%), 18 people with severe fatty liver (20.0\%), and 30 people without fatty liver (33.33\%). The water peak was near 4.7 parts per million (ppm), and the lipid peak was near $1.3 \mathrm{ppm}$. Analysis of variance revealed that differences in the lipid peak, the area under the lipid peak, ratio of the lipid peak to the water peak, and ratio of the area under the lipid peak to the area under the water peak were statistically significant among the groups. Area under the ROC curve (AUC) of area ratio in lipid and water and ratio in lipid and water, in no fatty liver group to mild fatty liver, mild fatty liver group to moderate fatty liver, and moderate fatty liver disease to severe fatty liver, were $0.705,0.900,0.975$. Therefore, $1 \mathrm{H}$ MRS is a noninvasive technique that can be used to provide information on the effect of liver steatosis on hepatic metabolic processes. This study indicates that the $1 \mathrm{H}$ MRS can be used as an indicator of steatosis in patients with chronic hepatitis C.
\end{abstract}

\section{Introduction}

Because of weight problems and insulin resistance in patients with nonalcoholic fatty liver disease (NAFLD), the prevalence of hepatic steatosis is growing quickly around the world [1,2]. Simple nonalcoholic steatosis can progress to more severe liver disease (nonalcoholic steatohepatitis [NASH] and cirrhosis), representing a menace to public health.

Liver biopsy continues to be the defacto standard for evaluating hepatic steatosis, despite well-established drawbacks regarding its invasiveness and sampling errors because of small sample sizes and inter-observer variability [3,4]. Noninvasive modalities for example ultrasound, computed tomography, and magnetic resonance imaging (MRI) happen to be useful for the assessment of hepatic steatosis [5-7]. However, these modalities don't particularly measure hepatic fat content, they're semiquantitative, plus they lack high sensitivity and specificity [8]. Numerous studies have centered on the function of imaging techniques as noninvasive options to liver biopsy for discovering and quantifying hepatic steatosis [9]. The reported sensitivities and specificities of various imaging techniques and various studies investigating exactly the same technique vary substantially.

$1 \mathrm{H}$ magnetic resonance spectroscopy (1H MRS) is really a safe and noninvasive alternative for quantifying hepatic fat content. The modality offers good reproducibility along with a detailed analysis of various liver lobes, and contains been evaluated in a variety of studies. The objective of this research ended up being to assess the need for $1 \mathrm{H}$ MRS in diagnosing hepatic steatosis in patients with NAFLD.

\section{Materials and Methods}

Patients. From January 2010 to June 2010, 90 patients with chronic hepatitis C were enrolled. The diagnosis of hepatitis C was based on the AASLD Clinical Guideline for Hepatitis C (2004). This study was conducted in accordance with the declaration of Helsinki. This study was conducted with 
approval from the Ethics Committee of Jilin University. Written informed consent was obtained from all participants.

According to the 2003 branch of the Chinese Medical Association to develop liver fatty liver disease classification criteria for grading liver fat content [19], 30-50\% hepatic steatosis was classified as mild fatty liver; $50-75 \%$ steatosis was categorized as moderate fatty liver, and greater than $75 \%$ steatosis was classified as severe fatty liver. Meanwhile, fatty degeneration of the field of vision affecting less than $30 \%$ of liver cells was classified as the absence of fatty liver. The severity of disease was scored according to the Ishak system. The classification of patients with mild and moderate disease was based on the Ishak fibrosis (F) and necroinflammatory (NI) scoring system as follows: mild hepatitis ( $\mathrm{F} \leq 2$ and $\mathrm{NI} \leq 3)$, moderate hepatitis $(3 \leq \mathrm{F}<6$ and $\mathrm{NI}>4)$, and cirrhosis ( $\mathrm{F}$ $=6)$. Liver disease was evaluated using 3.0T MRI 1H MRS. According to each area under the peak, we can calculate the percentage area and compare the values with those obtained via pathologic analysis to determine whether the aforementioned parameters differ among the groups.

\section{MRI and 1H MRS}

$1 \mathrm{H}$ MRS was performed with and without water suppression. Localized single voxel point resolved spectroscopy (PRESS) $\mathrm{BH}$ with TR/TE $=3000 \mathrm{~ms} / 35 \mathrm{~ms}$ and number of averages $=64$ were taken. A voxel of $2 \times 2 \times 2 \mathrm{~cm}^{3}$ was located mainly in the right parietal region of the liver in all subjects. Data acquisition was performed with breath holding to ensure that the scanning area of interest was constant and to reduce the impact of cardiac pulsatility. Liver tissue contains more water and fat, and the strongest $1 \mathrm{H}$ MRS signals detected are water and fat.

Statistical analysis. All statistical analyses were performed using SPSS 19.0 software (SPSS Inc., Chicago, IL). Mean data were analyzed using a t-test. All statistical tests were two-sided, and P $<0.05$ was considered statistically significant.

\section{Results}

Demographics and baseline characteristics. This group of patients included 26 (28.89\%), 16 (17.78\%), and 18 patients (20.0\%) with mild, moderate, and severe fatty liver, respectively, and 30 patients without fatty liver (33.33\%). In terms of gender differences, the proportion of males was higher than that of females in the moderate and severe fatty liver groups $(\mathrm{P}<0.05)$, whereas no gender differences were observed in the no fatty liver and mild fatty liver groups. In addition, total bilirubin(TBIL), Alanine aminotransferase(ALT), Aspartate transaminase (AST), triglyceride(TG), cholesterol, and fasting blood glucose levels were higher in the moderate and severe fatty liver groups than in the no fatty liver and mild fatty liver groups $(\mathrm{P}<0.05$, Table 1$)$.

Table 1 Patient demographics and baseline characteristics

\begin{tabular}{ccccc}
\hline & $\begin{array}{c}\text { No fatty } \\
\text { liver(n=30) }\end{array}$ & $\begin{array}{c}\text { Mild fatty } \\
\text { liver(n=26) }\end{array}$ & $\begin{array}{c}\text { Moderate fatty } \\
\text { liver(n=16) }\end{array}$ & $\begin{array}{c}\text { Severe fatty } \\
\text { liver(n=9) }\end{array}$ \\
\hline Age & $\begin{array}{c}49.8 \pm 10.6 \\
\text { Male 8 (53.33\%) }\end{array}$ & $\begin{array}{c}53.4 \pm 9.8 \\
\text { Male 6 (46.15\%) }\end{array}$ & $\begin{array}{c}52.7 \pm 10.4 \\
\text { Male 5 }(62.5 \%)^{*}\end{array}$ & $\begin{array}{c}55.6 \pm 11.2 \\
\text { Male 7 }(77.78 \%)^{*}\end{array}$ \\
HCnder & & & & \\
HCV-RNA(copies/ ml) & $5.18 \pm 1.20$ & $5.43 \pm 1.14$ & $5.23 \pm 1.65$ & $5.43 \pm 1.47$ \\
Child-Plug & $3 \pm 1$ & $4 \pm 1$ & $6 \pm 1$ & $7 \pm 1$ \\
TBIL & $12.7 \pm 6.7$ & $13.9 \pm 6.4$ & $26.6 \pm 6.9^{*}$ & $38.7 \pm 11.6^{*}$ \\
ALT & $12.9 \pm 10.6$ & $18.8 \pm 12.3$ & $62.4 \pm 13.2^{*}$ & $103.8 \pm 20.3^{*}$ \\
AST & $10.7 \pm 9.9$ & $16.5 \pm 10.1$ & $52.5 \pm 12.3^{*}$ & $87.7 \pm 16.2^{*}$ \\
TG & $1.2 \pm 0.3$ & $1.8 \pm 0.4$ & $3.2 \pm 1.1^{*}$ & $5.4 \pm 2.1^{*}$ \\
Chol & $2.7 \pm 1.2$ & $3.3 \pm 1.5$ & $5.4 \pm 1.8^{*}$ & $6.7 \pm 2.2^{*}$ \\
Blood glucose & $4.1 \pm 0.2$ & $4.3 \pm 0.3$ & $5.8 \pm 2.0^{*}$ & $7.4 \pm 3.3^{*}$ \\
\hline
\end{tabular}

${ }^{*} \mathrm{p}<0.05$ compare to no fatty liver goup and mild fatty liver group

Diagnosis of the severity of fatty liver. ROC curve of IH MRS paramenter is used to different the degree of fatty liver. the to compare no fatty liver group and mild fatty liver, area under the ROC 
curve (AUC) of area ratio in lipid and water and ratio in lipid and water were 0.705 and 0.71 , which havs certain reference significance, and the other parameters are not sensitive. And to compare mild fatty liver group and moderate fatty liver, area under the ROC curve (AUC) of area ratio in lipid and water and ratio in lipid and water were 0.900 and 0.780 , respectively, which has good sensitivity and specificity.

Different degree of fatty change $1 \mathrm{H}$ MRS peak ratio of fat and water, area ratio of fat water variance analysis.

Analysis peak ratio of fat and water, area ratio of fat water in patients with different degree of fatty liver, the results showed that peak ratio of fat and water, area ratio of fat water difference between groups were statistically significant $(\mathrm{P}<0.05)$.

\section{Discussion}

It's believed that roughly 3\% from the global human population is chronically have contracted HCV which roughly 4 million persons are recently infected every year. In 55-85\% of patients, HCV infection progresses to chronic liver disease, with lots of patients remaining asymptomatic. In roughly $20 \%$ of cases, fibrosis develops into cirrhosis, which results in hepatocellular cancer in fivePercent of patients every year $[13,14]$. Liver biopsy may be the reference standard for staging and grading chronic liver disease, however this invasive procedure isn't without risk. There's a minimal mortality rate and error rate connected with this particular procedure, predominantly because of undersampling, as typically under $1 / 50,000$ th from the liver volume is acquired for histologic evaluation [15]. Because of the issues connected with biopsy, a stable drive to recognize a highly effective noninvasive approach to evaluating liver damage has brought to developments in testing with serologic biomarkers of disease as well as in imaging. For ethical reasons and since most sufferers are reluctant to endure repeated procedures, treatment algorithms within the Uk rarely allow serial liver biopsy. The impetus to locate a reliable and repeatable biomarker of disease activity and reaction to treatment thus has restored focus [16].

MRS is really a valuable tool for that noninvasive assessment of metabolic processes in vivo. Due to the existence of certain compounds within the organization of nuclear protons, these compounds or metabolites produce certain chemical shifts in specific chemical environments. Small alterations in the magnetic resonance peak brought on by these changes might be collected with a magnetic resonance scanner and transformed into statistical spectra. Neuronal markers, membrane constituents, osmolytes, and also the energy status could be measured for detecting various illnesses and therapeutic monitoring in humans [17]. 1H MRS generates a spectrum of the several resonances of protons which are baked into different chemical bonds. Since the protons are encircled by various nuclei and electrons using their own magnetic qualities, small magnetic field perturbations exist in an organized manner, resulting in slight variations within the received frequencies of protons in various chemical bonds. Thus, caffeine shifts occur basically as a result of the variable electronegativity of adjacent chemical moieties within the molecule. Caffeine shift scale describes the positioning of resonances within the spectrum in parts per million, regardless of the area strength, in accordance with a reference set at parts per million. The actual frequency shift, however, measured in Hertz (Hz), is directly proportional to the effectiveness of the magnetic field, e.g., 1 parts per million from the proton spectrum at $1.5 \mathrm{~T}$ describes $64 \mathrm{~Hz}$ and also to $128 \mathrm{~Hz}$ at 3.0T. Therefore, with greater magnetic fields, the resonances be more effective separated. The regularity separation from the resonances or peaks describes the resolution from the spectrum [18].

The number of body fat peak (1.3 parts per million) towards the water peak (4.7 parts per million) is a very common meaning of the hepatic fat percentage as based on 1H MRS. By using this definition, Thomas et al. [19] reported the connection between body adiposity and steatosis in 11 patients with NASH and identified hepatic fat percentages as high as 75\%. However, inside a clinical study by Longo et al., a formula ([AUCtotal fat peaks/AUCtotal peaks]) for calculating hepatic fat content from $1 \mathrm{H}$ MR spectra was recommended. Exactly the same method was used in a sizable study by Szczepaniak et al., who evaluated the prevalence of hepatic steatosis in over 2300 participants from the Dallas Heart Study population [20]. 
In a nutshell, 3.0T 1H MRS might be a highly effective technology for assessing fat metabolic process in patients with chronic HCV. However, the research samples are relatively small, necessitating further in-depth exploration.

\section{References}

[1] Angulo, P., Current best treatment for non-alcoholic fatty liver disease. Expert Opin Pharmacother, 2003. 4(5): p.611-23. PMID:12739988, doi:10.1517/14656566.4.5.611

[2] Williams, R., Global challenges in liver disease. Hepatology, 2006. 44(3): p. 521-6. PMID:16941687, doi:10.1002/hep.21347

[3] Rector, R.S., J.P. Thyfault, Y. Wei, and J.A. Ibdah, Non-alcoholic fatty liver disease and the metabolic syndrome: an update. World J Gastroenterol, 2008. 14(2): p. 185-92. PMID:18186553, doi:

[4] Bashir, M.R., E.M. Merkle, A.D. Smith, and D.T. Boll, Hepatic MR imaging for in vivo differentiation of steatosis, iron deposition and combined storage disorder: single-ratio in/opposed phase analysis vs. dual-ratio Dixon discrimination. Eur J Radiol, 2012. 81(2): p. e101-9. PMID:21330083, doi: 10.1016/j.ejrad.2011.01.067

[5] Irimia, E., L. Mogoanta, I.O. Predescu, I.C. Efrem, C. Stanescu, L.A. Streba, and A.M. Georgescu, Liver steatosis associated with chronic hepatitis C. Rom J Morphol Embryol, 2014. 55(2): p. 351-6. PMID:24969985, doi:

[6] Rubbia-Brandt, L., P. Fabris, S. Paganin, G. Leandro, P.J. Male, E. Giostra, A. Carlotto, L. Bozzola, A. Smedile, and F. Negro, Steatosis affects chronic hepatitis C progression in a genotype specific way. Gut, 2004. 53(3): p. 406-12. PMID:14960525, doi:

[7] Cheng, F.K., D.M. Torres, and S.A. Harrison, Hepatitis C and lipid metabolism, hepatic steatosis, and NAFLD: still important in the era of direct acting antiviral therapy? J Viral Hepat, 2014. 21(1): p. 1-8. PMID:24329852, doi:10.1111/jvh.12172

[8] Moritou, Y., F. Ikeda, Y. Iwasaki, N. Baba, K. Takaguchi, T. Senoh, T. Nagano, Y. Takeuchi, T. Yasunaka, H. Ohnishi, Y. Miyake, A. Takaki, K. Nouso, and K. Yamamoto, Impact of comorbid hepatic steatosis on treatment of chronic hepatitis $C$ in Japanese patients and the relationship with genetic polymorphism of IL28B, PNPLA3 and LDL receptor. Acta Med Okayama, 2014. 68(1): p. 17-22. PMID:24553484, doi:

[9] Macaluso, F.S., M. Maida, C. Camma, G. Cabibbo, D. Cabibi, R. Alduino, V. Di Marco, A. Craxi, and S. Petta, Steatosis affects the performance of liver stiffness measurement for fibrosis assessment in patients with genotype 1 chronic hepatitis C. J Hepatol, 2014. 61(3): p. 523-9. PMID:24815874, doi: 10.1016/j.jhep.2014.04.045

[10] Ratziu, V., F. Charlotte, A. Heurtier, S. Gombert, P. Giral, E. Bruckert, A. Grimaldi, F. Capron, T. Poynard, and L.S. Group, Sampling variability of liver biopsy in nonalcoholic fatty liver disease. Gastroenterology, 2005. 128(7): p. 1898-906.PMID:15940625, doi:

[11] Mehta, S.R., E.L. Thomas, J.D. Bell, D.G. Johnston, and S.D. Taylor-Robinson, Non-invasive means of measuring hepatic fat content. World J Gastroenterol, 2008. 14(22): p. 3476-83. PMID:18567074, doi:

[12] Cho, C.S., S. Curran, L.H. Schwartz, D.A. Kooby, D.S. Klimstra, J. Shia, A. Munoz, Y. Fong, W.R. Jarnagin, R.P. DeMatteo, L.H. Blumgart, and M.I. D'Angelica, Preoperative radiographic assessment of hepatic steatosis with histologic correlation. J Am Coll Surg, 2008. 206(3): p. 480-8. PMID:18308219, doi: 10.1016/j.jamcollsurg.2007.08.020

[13] Bohte, A.E., J.R. van Werven, S. Bipat, and J. Stoker, the diagnostic accuracy of US, CT, MRI and 1H-MRS for the evaluation of hepatic steatosis compared with liver biopsy: a meta-analysis. Eur 
Radiol, 2011. 21(1): p. 87-97. PMID:20680289, doi:10.1007/s00330-010-1905-5

[14] Charatcharoenwitthaya, P. and K.D. Lindor, Role of radiologic modalities in the management of non-alcoholic steatohepatitis. Clin Liver Dis, 2007. 11(1): p. 37-54, viii. PMID:17544971, doi: 10.1016/j.cld.2007.02.014

[15] Bastati, N., D. Feier, A. Wibmer, S. Traussnigg, C. Balassy, D. Tamandl, H. Einspieler, F. Wrba, M. Trauner, C. Herold, and A. Ba-Ssalamah, Noninvasive differentiation of simple steatosis and steatohepatitis by using gadoxetic acid-enhanced MR imaging in patients with nonalcoholic fatty liver disease: a proof-of-concept study. Radiology, 2014. 271(3): p. 739-47. PMID:24576046, doi:10.1148/radiol.14131890

[16] Chabanova, E., D.S. Bille, E. Thisted, J.C. Holm, and H.S. Thomsen, (1) H MRS assessment of hepatic steatosis in overweight children and adolescents: comparison between $3 \mathrm{~T}$ and open $1 \mathrm{~T}$ MR-systems. Abdom Imaging, 2013. 38(2): p. 315-9. PMID:22736224, doi: 10.1007/s00261-012-9930-2

[17] Opstad, K.S., B.A. Bell, J.R. Griffiths, and F.A. Howe, an investigation of human brain tumour lipids by high-resolution magic angle spinning $1 \mathrm{H}$ MRS and histological analysis. NMR Biomed, 2008. 21(7): p. 677-85. PMID:18186027, doi:10.1002/nbm.1239

[18] Bischoff, M., [Importance of steatosis for MR diagnosis]. Rofo, 2013. 185(11): p. 1032-3. PMID:24308053, doi:

[19] Hwang, I., J.M. Lee, K.B. Lee, J.H. Yoon, B. Kiefer, J.K. Han, and B.I. Choi, Hepatic steatosis in living liver donor candidates: preoperative assessment by using breath-hold triple-echo MR imaging and 1H MR spectroscopy. Radiology, 2014. 271(3): p. 730-8. PMID: 24533869, doi: 10.1148/radiol.14130863

[20] Brook, G., V. Soriano, and C. Bergin, European guideline for the management of hepatitis B and $\mathrm{C}$ virus infections, 2010. Int J STD AIDS, 2010. 21(10): p. 669-78. PMID:21139144, doi:10.1258/ijsa.2010.010234 